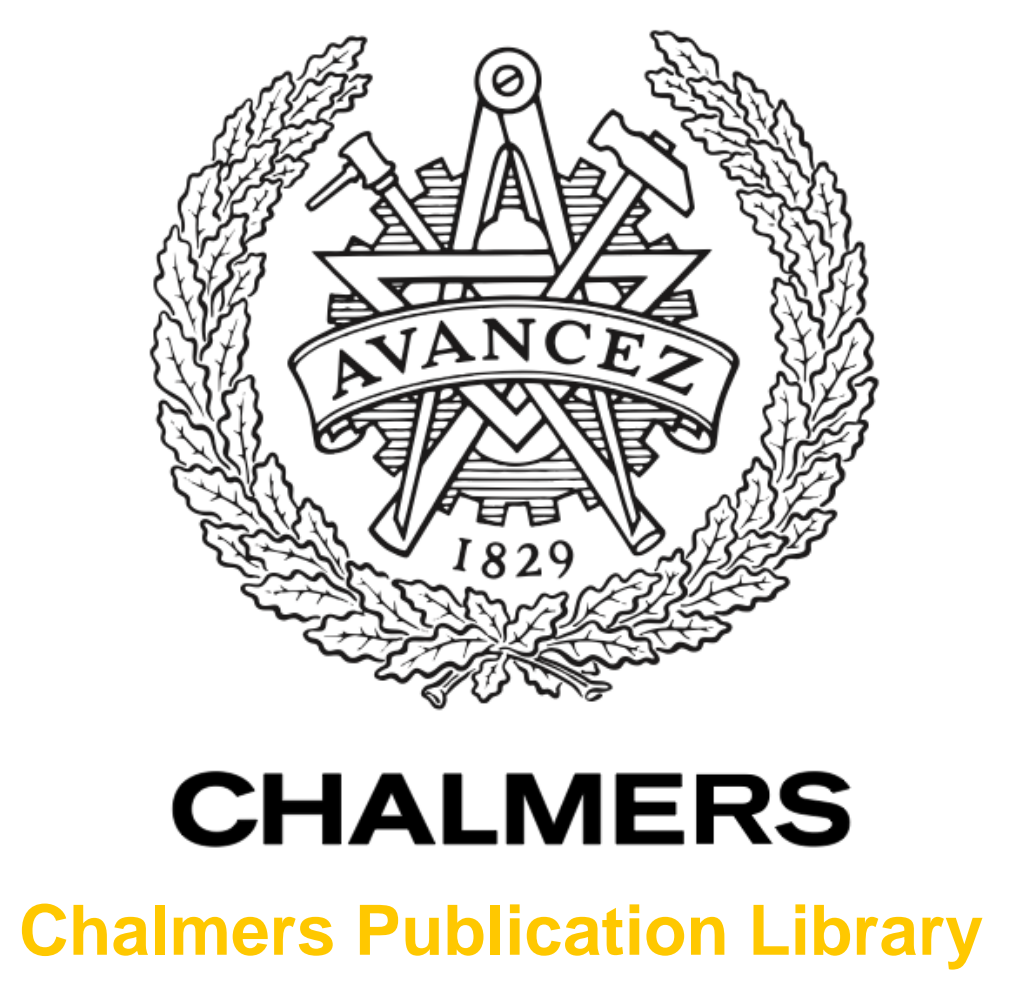

\title{
A novel dual-structure, self-healable, polysaccharide based hybrid nanogel for biomedical uses
}

This document has been downloaded from Chalmers Publication Library (CPL). It is the author's version of a work that was accepted for publication in:

Soft Matter (ISSN: 1744-683X)

Citation for the published paper:

Lin, L. ; Larsson, M. ; Liu, D. (2011) "A novel dual-structure, self-healable, polysaccharide based hybrid nanogel for biomedical uses". Soft Matter, vol. 7(12), pp. 5816-5825.

http://dx.doi.org/10.1039/c1sm05249g

Downloaded from: http://publications.lib.chalmers.se/publication/142342

Notice: Changes introduced as a result of publishing processes such as copy-editing and formatting may not be reflected in this document. For a definitive version of this work, please refer to the published source. Please note that access to the published version might require a subscription.

Chalmers Publication Library (CPL) offers the possibility of retrieving research publications produced at Chalmers University of Technology. It covers all types of publications: articles, dissertations, licentiate theses, masters theses, conference papers, reports etc. Since 2006 it is the official tool for Chalmers official publication statistics. To ensure that Chalmers research results are disseminated as widely as possible, an Open Access Policy has been adopted.

The CPL service is administrated and maintained by Chalmers Library. 


\title{
A novel dual-structure, self-healable, polysaccharide based hybrid nanogel for biomedical uses
}

\author{
Li-Jih Lin, ${ }^{a}{ }^{\ddagger}$ Mikael Larsson ${ }^{a, b}$ and Dean-Mo Liu ${ }^{* a}$ \\ Received (in $X X X, X X X)$ Xth $X X X X X X X X X 20 X X$, Accepted Xth $X X X X X X X X X 20 X X$ \\ ${ }_{5}$ DOI: 10.1039/b000000x
}

\begin{abstract}
A new unique dual-structure hydrogel composed of nanostructures of amphiphilic chitosan (CHC) dispersed in a sodium alginate matrix (SAL) is presented. The successful creation of the composite is based on combining chitosan and sodium alginate without precipitation or agglomeration, which has not been previously reported. The CHC/SAL composite gels presents a number of properties making them 10 attractive for biomedical applications, in particular as implantable depot gels or in dermal applications. The gels are shown to form rapidly upon exposure of the combination solution to $\mathrm{Ca}^{2+}$ containing gelation medium. The formed gels have storage moduli similar to soft tissue and displays shear reversible gelation with fast recovery of mechanical properties, in addition to self healing capability at certain compositions. The gels exhibit moderate swelling in deionized water and low swelling in simulated body fluid and cell 15 culture media. The drug release from the composite gels is demonstrated using the hydrophobic drug alltrans retinoic acid, which is used in cancer and skin disorder therapies. The drug release initially occurs through a Fickian mechanism for a fraction of the loaded drug, where the fraction released during this process depends on release media and gel composition. A large fraction of loaded drug can be retained for long term depot drug delivery. Furthermore, the CHC/SAL gels are determined to have low toxicity and 20 skin irritation.
\end{abstract}

\section{Introduction}

With the rapidly evolving knowledge in the biomedical field of today there is increasing demand for materials that can meet the needs of new applications. One specific application is depot 25 systems for sustained drug delivery. Depot systems hold the benefit of providing sustained drug release over long times, as well as being able to provide local therapeutic effect. ${ }^{1-3}$ There are several challenges in the design of materials to be used in such systems. The materials should allow for high loading of 30 hydrophobic drugs and control over the release process. ${ }^{4}$ In the case of implantable depot systems the administration should be easy, as for injectable in vivo gelling formulations. Furthermore, the materials should be biocompatible in their given application. Obviously, the materials used should be non toxic and non irritant 35 for transdermal applications. For implantable devices the mechanical properties of the device are also of great importance, it has been stated that "the mechanical property of the interface between an implant and its surrounding tissues is critical for the host response and the performance of the device". 5

40 One way to overcome the low solubility of hydrophobic drugs is to make use of amphiphilic copolymers or modified polymer micelles, such polymer materials tend to self-assemble into nanoscale micelle-like structures, having core-shell architecture in aqueous solution. ${ }^{6-8}$ This structure provides the ability to 45 encapsulate and release hydrophobic compounds. In addition, such drug carriers can commonly be designed to be biocompatible and/or biodegradable. ${ }^{6}, 9,10$ This kind of nano carriers can be effectively utilized in designing hydrogel systems for release of drugs with low solubility. Gou et al. presented the 50 idea to combine a nanoscale carrier and a hydrogel matrix into a composite dual structure delivery system for hydrophobic drug release, ${ }^{11}$ inspired by similar reports on micro- and nano-particles in thermo-sensitive hydrogel composite drug delivery systems. ${ }^{12}$ 13

55 The mechanical properties, and in particular the storage modulus, of a gel matrix is dependent on the crosslink density. ${ }^{14}$ However, for composites where particle additives are dispersed in a gel network the mechanical properties can be significantly different from the pure matrix, depending on additive 60 concentration, modulus and the extent of additive-matrix interaction. ${ }^{15-17}$ When the additives are hard fillers two ultimate cases can be discriminated: ${ }^{18}$ (1) No interaction between the dispersed particles; this causes a decrease in gel modulus with increasing polymer volume fraction. (2) A strong interaction 65 between the fillers and the matrix; this causes an increase in modulus of the gel with the increasing polymer volume fraction if the filler material is harder than the gel matrix. For additives having sizes similar to the matrix network the effects are more complex, and aggregation of the additive is an important factor. ${ }^{19}$ 70 Nanofillers having strong interaction with the matrix have been reported to increase the storage modulus of the composite far more than expected from traditional filler theory. ${ }^{20,21}$ On the other hand, alginate gels prepared using glycerol or low 
molecular weight dextran as additives displayed an increased viscosity in the gel liquid phase but did not show any changes in storage modulus. However, for high molecular weight dextran the storage modulus was significantly lowered, ${ }^{22,} 23$ this was 5 explained by that the high molecular weight dextran disturbed the crosslink structure of the alginate.

Alginate and chitosan are two natural polymers that are biodegradable, biocompatible, non-toxic, and mucoadhesive. ${ }^{24}$ Because of those desirable properties they are commonly used 10 and have great future potential in biomedical applications, such as; drug delivery systems, tissue engineering scaffolds, and in food industry as stabilizers, thickeners and gelling agents. ${ }^{3,}$ 24-30 Alginate is a linear block copolymer composed of homopolymeric blocks of (1-4) linked $\alpha$-L-guluronate $(G)$ and $\beta$ 15 D-mannuronate $(\mathrm{M})$ residues. $^{24,31,32}$ The relative number of Mand G-blocks depends on the origin of alginate. One of the most important properties of alginate, with regard to biomedical applications, is the ability to form gels by interaction with divalent cations such as $\mathrm{Ca}^{2+}$. The gelation and cross-linking of 20 the alginate is mainly achieved by interaction between the carboxyl groups and the divalent cations, and the stacking of these G-blocks to form the characteristic egg-box structure. ${ }^{24,}$ 33-35 In addition, the highly swelling sodium alginate form (SAL) also has the ability to form gels by the exchange of sodium ions from 25 the G-blocks with divalent cations. Amphiphilically modified chitosan, named carboxymethyl-hexanoyl chitosan (CHC), has previously been synthesized in an aqueous system without the aid of surfactants, organic solvents, emulsion phases, or template cores, to form a hollow nanocapsules in water. ${ }^{27,} 28,36$ The CHC 30 has excellent encapsulating efficiency for hydrophobic drugs due to its self-assembly properties and hydrophobic domains.

In this study, amphiphilic chitosan was synthesized and used as a hydrophobic drug carrier in an alginate hydrogel matrix. Composite gels of sodium alginate and micelle-like amphiphilic 35 carboxymethyl-hexanoyl chitosan nanoparticles were prepared in various compositions, varying the SAL and CHC content, the amount of glycerol in the gel forming solution and the amount of calcium chloride in the gelation media. The composite gels were characterized with regard to a number of properties such as; 40 gelation time, equilibrium swelling, rheological properties, selfhealing behaviour and release of the hydrophobic drug all-trans retinoic acid. The dependences of the gel properties on the compositions of the hydrogels were then discussed.

\section{Materials and methods}

\section{${ }_{45}$ Materials}

2-propanol, hexanoyl anhydride, all-trans-Retinoic acid, Chitosan $(\mathrm{Mw}=215000 \mathrm{~g} / \mathrm{mol}$, deacetylation degree $=85-90 \%)$, chloroacetic acid, sodium alginate (low viscosity, $250 \mathrm{cps}$ for $2 \%$ at $25^{\circ} \mathrm{C}$ ) and sodium hydroxide was purchased from Sigma${ }_{50}$ Aldrich. Calcium chloride was acquired from Showa. Glycerol was purchased from Riedel-de Haën. Minimal essential medium $(\alpha-\mathrm{MEM})$ was prepared with components bought from Gibco and $1.5 \mathrm{x}$ simulated body fluid (1.5BF) was prepared in-house.

Preparation of amphiphilic chitosan/sodium alginate 55 combination solutions

Amphiphilic chitosan (CHC) was prepared through both hydrophilic carboxymethyl and hydrophobic hexanoyl substitutions, as previously described 27,28 . CHC solution (2\% $\mathrm{w} / \mathrm{w})$ was prepared in a vial, using sodium hydroxide solution to 60 adjust the $p H$ value to slightly alkaline $(p H=7.5-8.5)$. Sodium Alginate (SAL) solutions were prepared with different concentrations $(1,2,3$, and $4 \% \mathrm{w} / \mathrm{w})$. CHC/SAL combination solutions with different compositions were formed by mixing $\mathrm{CHC}$ solution containing varying amount of glycerol $(0,10$, or 20 ${ }_{65} \% \mathrm{w} / \mathrm{w}$ ) with an equal volume of SAL solution.

\section{Hydrogel formation}

The composite hydrogels were formed in the presence of various calcium ion concentrations as follows; $\mathrm{CHC} / \mathrm{SAL}$ combination solution $(2 \mathrm{ml})$ in a glass Petri dish was submerged into calcium 70 chloride solution $(50 \mathrm{ml}, 1,2$, or $3 \% \mathrm{w} / \mathrm{w})$ at room temperature. The time to form a gel (designated as gelation time) was determined using a vial tilting method, where no flow within 1 minute of inverting the vial was the criterion for gel state ${ }^{37,38}$.

\section{Rheological measurements}

75 Rheological characterization of the CHC/SAL composite hydrogels was performed on a strain-controlled rheometer (Rheological Scientific, ARES instrument) using parallel-plate fixture. Olive oil was used to cover the surface of the composite hydrogels in order to avoid water evaporation during the analysis. ${ }_{80}$ The rheological properties of the CHC/SAL composite hydrogels were characterized by strain sweep tests $(\gamma=0.01 \sim 100 \%)$ and small deformation tests $(\gamma=0.015 \sim 7 \%)$ using a fixed frequency $(\omega=10 \mathrm{rad} / \mathrm{s})$ and a temperature of $37{ }^{\circ} \mathrm{C}$. The gap at the apex of the parallel-plate was set to be $2 \mathrm{~mm}$ and samples were placed 85 between the parallel-plate and the platform. To investigate the recovery properties of the samples after exposure to high shear strain, the following program was applied: $\gamma=0.1 \%(100 \mathrm{~s}) \rightarrow \gamma$ $=100 \%(100 \mathrm{~s}) \rightarrow \gamma=0.1 \%(200 \mathrm{~s}) \rightarrow \gamma=100 \%(200 \mathrm{~s}) \rightarrow \gamma=$ $0.1 \%(300 \mathrm{~s})$.

\section{${ }_{90}$ Investigation of self-healing capability}

The self-healing capability of the composite hydrogels was investigated as follows; two types of samples were prepared, one was coloured by Trypan blue and the other was a pure CHC/SAL hydrogel. The samples were cut into a size of $3 \times 1 \times 0.5 \mathrm{~cm}$ and 95 the freshly produced surfaces of two samples with different colour were brought together within one minute. After allowing healing to proceed for 30 minutes to one hour, the healed composite hydrogel bridge was suspended horizontally and vertically.

\section{Equilibrium Swelling}

To determine the equilibrium swelling under different conditions, gels made from CHC/SAL (about $2 \mathrm{~g}$ ) were lyophilized and weighted $\left(\mathrm{W}_{\mathrm{d}}\right)$. The dried hydrogels were immersed in di-water, medium $(\alpha-\mathrm{MEM}+10 \% \mathrm{FBS})$, or $1.5 \mathrm{SBF}$ for 1 day until 105 equilibrium swelling state had been attained. After removal of water from the surface of the swollen hydrogels, the samples were weighted $\left(\mathrm{W}_{\mathrm{s}}\right)$. The equilibrium swelling degree (ESD) was calculated using the following equation:

$$
\operatorname{ESD}=\left(W_{s}-W_{d}\right) / W_{d}
$$


Retinoic acid released from varying formulations of $\mathrm{CHC} / \mathrm{SAL}$ composite hydrogels was determined in different release environments. Drug loaded gels were prepared as follows; a stock solution of retinoic acid was prepared by dissolving of retinoic 5 acid $(100 \mathrm{mg})$ in isopropanol $(50 \mathrm{ml})$. The stock solution was diluted to achieve the final retinoic acid concentration $(100 \mu \mathrm{g} /$ $\mathrm{ml}$ ), subsequently $\mathrm{CHC}$ was added (2 \% w/w). Composite hydrogels with different compositions were then prepared as described above. To investigate the release profiles for the drug10 loaded CHC/SAL composite hydrogels, samples were submerged in di-water or $1.5 \mathrm{SBF}(3 \mathrm{ml})$. At predetermined times samples were extracted $(1 \mathrm{ml})$ and centrifuged at $12000 \mathrm{rpm}$ for $5 \mathrm{~min}$. Subsequently, the drug concentration in the supernatant was determined from the absorbance at $340 \mathrm{~nm}$ using a UV-Vis 15 spectrophotometer (Evolution 300, Thermo scientific). The extracted volume was replaced with an equal volume of fresh dissolution medium, which was accounted for in the release calculations.

\section{Cytotoxicity Assay}

${ }_{20}$ WS1 human fetal skin fibroblast cell lines (BCRC number: 60300) were grown in minimum essential medium (MEM) with FBS $(10 \%)$, non-essential amino acids $(0.1 \mathrm{mM})$, and sodium pyruvate $(1.0 \mathrm{mM})$. The cells were incubated at $37{ }^{\circ} \mathrm{C}$, in a $\mathrm{CO}_{2}$ containing $(5 \%)$ humidified atmosphere. The culture medium

25 was changed every two to three days. For all experiments, cells were harvested from sub-confluent cultures using trypsin and were re-suspended in fresh complete medium before plating. To investigate the in vitro cytotoxicity of the $\mathrm{CHC} / \mathrm{SAL}$ composite gel, viability of human WS1 fetal skin fibroblasts was analyzed 30 with the MTT assay. CHC/SAL gels were formed from combination solution $(0.5 \mathrm{ml})$ with the weight ratios $\mathrm{CHC} / \mathrm{SAL} /$ glycerol $=1 / 1.5 / 0$ prepared by exposure to $\mathrm{CaCl}_{2}(2 \%$ w/w). Two kinds of samples, one loaded with retinoic acid (100 $\mu \mathrm{g} / \mathrm{ml}$ ) and one without drug (pure gel), were prepared in 2435 well plates. Briefly, $3 \times 10^{4}$ cells were plated to allow the cells to attach at $37{ }^{\circ} \mathrm{C}$ in an atmosphere with $\mathrm{CO}_{2}(5 \%)$. After 1 and 2 days incubation, MTT/medium (1:9) combination solution (100 $\mu \mathrm{l})$ was added and incubation was continued for another 4 hours. Then, DMSO was added to solve the precipitate, which formed 40 from the reaction between MTT reagents and live cells, and the solution was transferred to a 96-well plate. The result solution absorbance values were determined at $595 \mathrm{~nm}$ using a Sunrise absorbance microplate reader (DV990/BV4, GDV Programmable MPT Reader).

\section{${ }_{45}$ In vivo skin irritation test}

The Draize model and its modification such as "UNI EN ISO10993-10:1996" and "USP Biological Tests" are generally used to examine the degree of skin primary irritation utilizing healthy rabbits ${ }^{39-41}$. Following the Draize model, the back of ${ }_{50}$ healthy male New Zealand white rabbits were narrowly clipped free of fur with an electric clipper 4 hours before application of samples. Each rabbit $(n=6)$ received six parallel epidermal abrasions with a sterile needle $(26 \mathrm{G} 1 / 20.45 \times 13 \mathrm{~mm})$ at one test site while the skin at the opposite site remained intact. Samples 55 were prepared by coating gel $(0.5 \mathrm{ml}$, weight ratios $\mathrm{CHC} / \mathrm{SAL} /$ glycerol $=1 / 0.5 / 0$ or $1 / 1.5 / 0$, prepared by exposure to $\left.2 \% \mathrm{w} / \mathrm{w} \mathrm{CaCl}_{2}\right)$ on fixed size gauzes with 1 " $\times 1$ " $(2.54 \times 2.54$ $\mathrm{cm})$ square. The patches were covered with a non-reactive tape and the entire test site was swathed with a non-occlusive 60 bandage. After a 24 hour treatment, the bandage and gauze sample were removed. The test sites were swabbed with physiological saline solution to remove any remaining test article residue. The used evaluation procedure was the one adopted in the U.S. Federal Hazardous Substance Act (FHSA) ${ }^{42}$, and is 65 described in supporting information.

At times 24, 48, and 72 hours after sample application, the test sites were evaluated for dermal reactions, defined as erythema and edema, according to the Draize - FHSA scoring system (supporting information). The score of primary irritation of the 70 test was calculated for various dosages. The Primary Irritation Index (PII) was calculated as the arithmetical mean ${ }^{40,41}$.

$$
\mathrm{PII}=\frac{\sum \text { Erythema and Edema grade at } 24,48 \text { and } 72 \mathrm{~h}}{\text { Number of observations }}
$$

and the evaluation of PII was performed according to Table 1.

Table 1 Evaluation of Primary Irritation Index

\begin{tabular}{cc}
\hline Index & Evaluation \\
\hline 0.00 & No irritation \\
$0.04-0.99$ & Irritation barely perceptible \\
$1.00-1.99$ & Slight irritation \\
$2.00-2.99$ & Mild irritation \\
$3.00-5.99$ & Moderate irritation \\
$6.00-8.00$ & Severe irritation \\
& \\
\hline
\end{tabular}

\section{Results and discussion}

To the authors' knowledge, there has to date been no reported method to provide a steady combination solution with chitosan and sodium alginate. This is owing to two main reasons: (1) the 80 opposite charge of the polymers leads to electrostatic attraction promoting aggregation; (2) the property that sodium alginate (SAL) forms an acid gel by the addition of acidic chitosan solution. ${ }^{24}$ Here, amphiphilic carboxymethyl-hexanoyl chitosan (CHC) was used because of its proven potential as a hydrophobic ${ }_{85}$ drug carrier, utilizing self-assembly. ${ }^{27}$ To prevent aggregation and acid gel formation upon combination of the CHC and SAL solutions the $\mathrm{CHC}$ solution was adjusted to slightly alkaline ( $p H=7.5 \sim 8$ ) prior to mixing. Ordinarily, an acidic chitosan solution would rapidly produce a hydrated precipitate upon 90 addition of a strong base, such as $\mathrm{NaOH}$. This is due to the reduced positive charge density along the chitosan chains $\left(\mathrm{NH}_{3}{ }^{+}\right)$. 


\section{Soft Matter}

www.rsc.org/xxxxxx

Paper
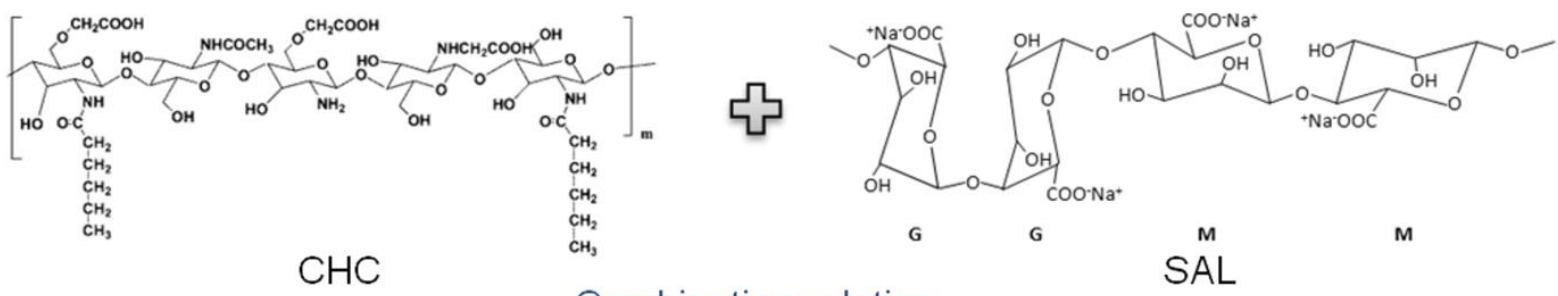

\section{Combination solution}

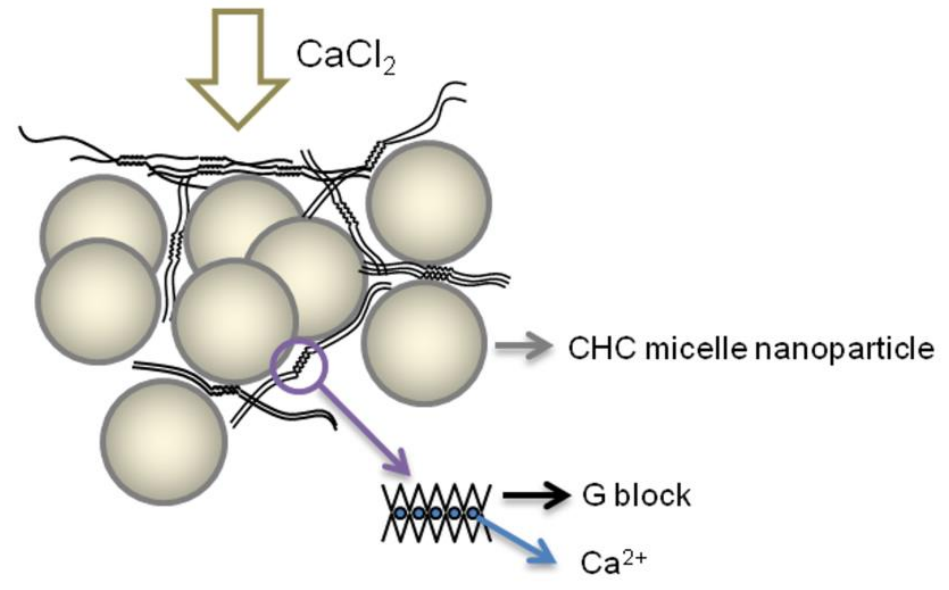

Scheme 1 Molecular structure of modified amphiphilic chitosan (CHC) and sodium alginate (SAL) and the suggested crosslinked network structure after gelation by $\mathrm{CaCl}_{2}$ rich medium.

The neutral chains would interact strongly through hydrogen 5 bonding and hydrophobic interactions between chains. There was no hydrated precipitate but only a little agglomerate when the amphiphilic CHC solution was adjusted to slightly alkaline by addition of $\mathrm{NaOH}$. This because of the steric effect from the long hexanoyl groups prevents hydrogen bonding between $\mathrm{CHC}$ 10 chains, ${ }^{43}$ as shown in Scheme 1, and because the self-assembly property into a micelle structure may further reduce the aggregation tendency. At the set $\mathrm{pH}$ value, the zeta potential of the CHC solution would be close to neutral, as the isoelectric point was determined to be about 7.5. The fact that the modified 15 chitosan is neutrally charged, and soluble at slightly alkaline $p H$, allows for the successful combination with sodium alginate. Indeed, no aggregation or gelation was observed upon forming the $\mathrm{CHC} / \mathrm{SAL}$ combination solution.

Below the properties of hydrogels prepared from CHA/SAL 20 combination solutions with different compositions is discussed with special focus on realistic biomedical applications.

\section{Hydrogel formation}

SAL is well known to form a strong gel upon exposure to calcium ions. The calcium cross-links the alginate chains by replacing 25 sodium ions with calcium ions to form the well known "egg box" structure through electrostatic forces between guluronic groups expressed on different alginate chains and bridging calcium ions. $^{22}$ Therefore, gelation of the $\mathrm{CHC} / \mathrm{SAL}$ combination solutions was induced by exposure to $\mathrm{CaCl}_{2}$ solution (gelation 30 media). The $\mathrm{CHC}$ concentration in the combination solution was above the aqueous critical aggregation concentration, as such the CHC should exist in self assembled micelle like structures, having diameters in the range $50-200 \mathrm{~nm} \cdot{ }^{36,44}$ Thus the structure of the formed composite gels is proposed to be a crosslinked 35 alginate matrix with embedded nano micelles. Scheme 1 shows the structure of $\mathrm{CHC}$ and SAL, as well as schematic drawing of the proposed composite gel structure.

The gel formation process and the gelation rate of the composite hydrogels were observed at room temperature. The 40 gelation time could be adjusted by varying $\mathrm{SAL}$ and $\mathrm{CaCl}_{2}$ concentration, with the fastest gelation time being close to instantaneous and the longest being about $10 \mathrm{~s}$ (Table 2). As an example, the gel with the weight ratios $\mathrm{CHC} / \mathrm{SAL} /$ glycerol $=$ $1 / 1 / 5$, prepared by exposure to $1 \%$ w/w $\mathrm{CaCl}_{2}$ solution formed a 45 gel in roughly $10 \mathrm{~s}$.

Table 2 Gelation time (s) depending on SAL concentration in the combination solution and $\mathrm{Ca}^{2+}$ concentration in the gelation medium.

\begin{tabular}{lccc}
\hline & $1 \% \mathrm{SAL}$ & $1.5 \% \mathrm{SAL}$ & $2 \% \mathrm{SAL}$ \\
\hline $1 \% \mathrm{Ca}^{2+}$ & 10 & 5 & 3 \\
$2 \% \mathrm{Ca}^{2+}$ & 3 & $<1$ & $<1$ \\
$3 \% \mathrm{Ca}^{2+}$ & 1 & $<1$ & $<1$ \\
& & & \\
\hline
\end{tabular}



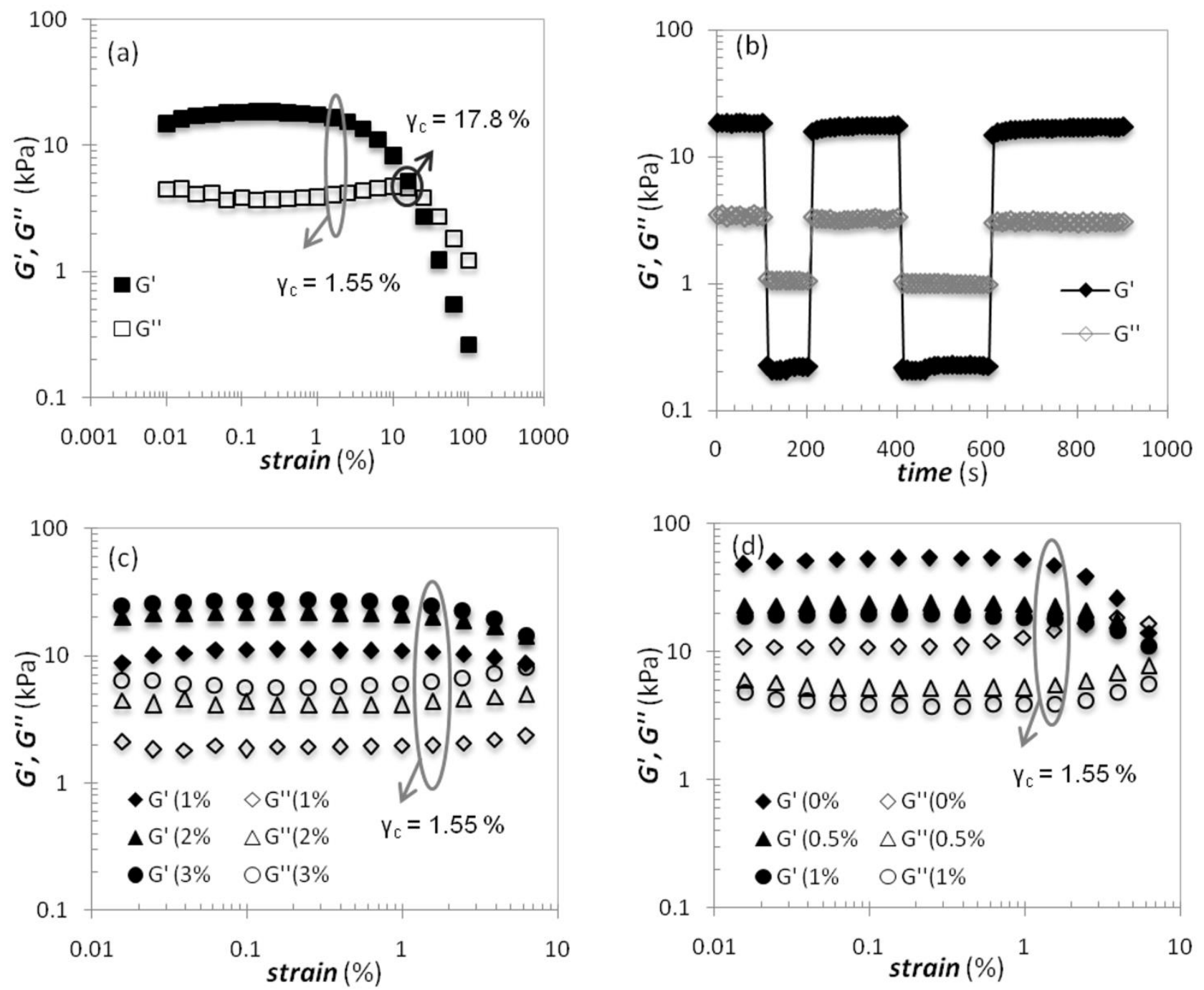

Fig. 1 Rheological properties of CHC/SAL composite hydrogels. (a) Large strain sweep $(\gamma=0.01 \sim 100 \%$ ) for gels having weight ratios $\mathrm{CHC} / \mathrm{SAL} /$ glycerol $=1 / 1.5 / 0$ prepared by exposure to $3 \% \mathrm{w} / \mathrm{w} \mathrm{CaCl}_{2}$. (b) Continuous step strain measurement $(\gamma=0.1$ and $100 \%)$ for hydrogel with the weight ratios $\mathrm{CHC} / \mathrm{SAL} /$ glycerol $=1 / 1.5 / 0$. Small deformation tests $(\gamma=0.015 \sim 7 \%)$ for samples with weight ratios; $(\mathrm{c}) \mathrm{CHC} / \mathrm{SAL} / \mathrm{glycerol}=1 / 1.5 / 5$ 5 prepared using varying $\mathrm{CaCl}_{2}$ concentration in the gelation medium and (d) $\mathrm{SAL} /$ glycerol $=1.5 / 0$ having varying $\mathrm{CHC}$ content, prepared by exposure to 2 $\% \mathrm{w} / \mathrm{w} \mathrm{CaCl}_{2}$.

By increasing the SAL concentration from 1 to $2 \%$ w/w, keeping the other conditions constant, the gelation time displayed an obvious decrease from 10 to $3 \mathrm{~s}$. With increasing ratios of $\mathrm{Ca}^{2+}$ in 10 the gelation media, the gelation time was greatly decreased. In fact, the gelation appeared to occur almost immediately for all but the samples with the lowest SAL concentration. The extremely quick gelling makes the CHC/SAL system a great candidate for injectable gel applications, where the combination solution could 15 be co-injected with calcium containing gelation medium

\section{Rheological properties}

The rheological properties of hydrogels are of great importance in determining their performance in different applications and under different conditions; in addition it gives information about the 20 structure of the gels. CHC/SAL composite hydrogels were subjected to strain sweep tests, monitoring the storage $\left(G^{\prime}\right)$ and loss modulus $(G$ "). As shown in Fig. 1a, there was a gel-liquid transition point $\left(\tan \delta=G^{\prime} / G^{\prime \prime}=1 ; \gamma_{\mathrm{g}}=17.8 \%\right)$ indicating a breakdown of the gel state to a quasi-liquid state above a 25 threshold strain. Reversible shear induced breakdown has previously been reported for pure alginate ${ }^{32}$ and chitosan ${ }^{45}$ gels. To investigate if this property was retained for $\mathrm{CHC}$ containing gels the sample with the weight ratios $\mathrm{CHC} / \mathrm{SAL} /$ glycerol = $1 / 1.5 / 0$, prepared by exposure to $3 \%$ w/w $\mathrm{CaCl}_{2}$, was 
investigated for shear reversibility. It was found to rapidly recover to its original gel state after a high shear strain induced structural breakdown, as shown in Fig. 1b. When high shear strain was applied, with the corresponding high shear stress $(\gamma=$ $5100 \%)$ and $(\omega=10 \mathrm{rad} / \mathrm{s})$, the $G$ ' values decreased from $18 \mathrm{kPa}$ to $0.21 \mathrm{kPa}$ resulting in a quasi-liquid state $(\tan \delta \approx 5)$. However, when the strain amplitude was decreased $(\gamma=0.01 \%)$ at the same frequency, $G$ ' instantly recovered its initial value and the system returned to a quasi-solid (gel) state ( $\tan \delta \approx 0.2$ ). In fact, the shear 10 recovery of the composite gels was extremely fast compared to pure alginate ${ }^{32,46}$ and chitosan ${ }^{45}$ gels, for which the reported shear recovery occur over a longer period of time.

In Fig. 1c, the effect of calcium chloride concentration in the gelation medium $(1,2$, and $3 \% \mathrm{w} / \mathrm{w})$ is shown for samples with 15 the weight ratios $\mathrm{CHC} / \mathrm{SAL} /$ glycerol $=1 / 1.5 / 5$, as determined by small deformation test. All samples displayed a plateau region in their moduli below the same critical strain $\left(\gamma_{c}\right)$ at which the polymeric system starts to display nonlinear viscoelastic behaviour (gel structure breaks up). ${ }^{45}$ When the strain was larger 20 than $\gamma_{c}$, there was a rapid decrease of the storage modulus. The fact that all sample preparations displayed $G$ ' values larger than $G$ " at small strains clearly proves the gel character under those conditions. The results show that $G^{\prime}$ and $G$ " values increased with the increasing concentration of $\mathrm{CaCl}_{2}$ in the gelation 25 medium. The modulus values of hydrogels prepared using $3 \%$ w/w $\mathrm{CaCl}_{2}$ in the gelation medium was almost about 1.2 times larger than using $2 \% \mathrm{w} / \mathrm{w} \mathrm{CaCl}_{2}$ and 2.4 times larger than using $1 \% \mathrm{w} / \mathrm{w} \mathrm{CaCl}_{2}$. According to rubber elasticity theory the correlation between storage modulus and the network crosslink 30 density can be described by the equation: ${ }^{47}$

$$
G=g R T N
$$

where $G$ is the network equilibrium shear modulus; $g$ is a constant, nearing 1.0 for incompressible materials; $R$ is the gas constant; $T$ is the absolute temperature; $N$ is the number of 35 elastically active network chains per unit volume for a network. Although this Eq. 3 is derived for networks of Gaussian chains, it can be applied to real polymer materials to provide an indication of network structure from shear modulus behaviour. ${ }^{48,}{ }^{49}$ Segeren et al. found several features of alginate gels formed by $\mathrm{Ca}^{2+}$ to be 40 consistent with rubber elasticity theory. ${ }^{50}$ Thus, it can be concluded that increasing $\mathrm{Ca}^{2+}$ concentration in gelation medium resulted in a rise of crosslinking density with the corresponding increase in storage modulus.

In this study, it was also crucial to elucidate the role of $\mathrm{CHC}$ 45 nanoparticles in determining the gels properties, in addition to providing a platform for delivery. As seen from Fig. 1d the presence of $\mathrm{CHC}$ nanoparticles decreased the storage modulus compared to pure alginate gels. It is known that particle additives alter the storage modulus depending on the interactions between 50 additive and network chains ${ }^{18,51}$ For calcium alginate gels Zhang et al. found that the addition of low molecular weight $(M W)$ dextran or glycerol prior to gelation had no significant effect on storage modulus. In contrast, gels to which high $M W$ dextran was added displayed a decrease of the storage modulus, as compared 55 to pure calcium alginate gels. ${ }^{22}$ This was explained by the steric effects of the high $M W$ dextran disturbing the crosslinking structure of the alginate gels. The same explanation seems
Table 3 Critical strain and cohesion energies for various preparations of CHC/SAL hydrogels (weight ratio $=1 / 1.5$ ).

\begin{tabular}{ccc|ccc}
\hline Glycerol $(\%)^{\mathrm{a}}$ & $\gamma_{\mathrm{c}}(\%)$ & $E_{c}\left(\mathrm{~kJ} / \mathrm{m}^{3}\right)^{\mathrm{b}}$ & $\mathrm{Ca}^{2+}(\%)^{\mathrm{c}}$ & $\gamma_{\mathrm{c}}(\%)$ & $E_{c}\left(\mathrm{~kJ} / \mathrm{m}^{3}\right)^{\mathrm{b}}$ \\
\hline 0 & 1.55 & $20.1 \pm 0.06$ & 1 & 1.55 & $13.3 \pm 0.07$ \\
5 & 1.55 & $29.7 \pm 0.05$ & 2 & 1.55 & $24.2 \pm 0.08$ \\
10 & 1.55 & $35.7 \pm 0.04$ & 3 & 1.55 & $29.7 \pm 0.05$
\end{tabular}

${ }_{60}{ }^{a}$ Percent glycerol in the combination solution, gels prepared by exposure to gelation medium with $3 \% \mathrm{CaCl}_{2} .{ }^{\mathrm{b}} \pm$ indicates maximum deviation from mean. ${ }^{\mathrm{c}} \mathrm{The} \mathrm{Ca}^{2+}$ percent is concentration in gelation medium, the combination solution contained $5 \%$ glycerol.

plausible for the lower storage modulus of the composite gels in 65 this study. The $\mathrm{CHC}$ nanoparticles inside the gel structure would appear as steric hindrances, separating the alginate chains, resulting in decreased crosslink density with the associated decrease of storage modulus.

Interestingly, it was found that the presence of glycerol in the ${ }_{70} \mathrm{CHC} / \mathrm{SAL}$ composites increased the storage modulus. This is in contrast to what has previously been reported for pure calcium alginate gels. ${ }^{22}$ Small deformation tests for samples prepared with varying glycerol contents (fixed weight ratios $\mathrm{CHC} / \mathrm{SAL}=1 / 1.5$ and $3 \% \mathrm{w} / \mathrm{w} \mathrm{CaCl}_{2}$ in the gelation medium) revealed that the $G$,

75 values for samples prepared with $10 \%$ glycerol was 1.2 times larger than if prepared with $5 \%$ glycerol and 1.75 times larger than gels prepared without glycerol (See Supplementary material, comparisons made at $\gamma=0.245 \%$ ). It seems likely that in the composite gels the glycerol act as a hydrogen bonding connector 80 between the otherwise non-interacting $\mathrm{CHC}$ nanoparticles and alginate chains, as well as between $\mathrm{CHC}$ nanoparticles. This hydrogen bridging should act crosslinking, and thus increase the storage modulus of the gels.

All of the investigated gel formulations displayed the same 85 critical strain, which together with the storage modulus can be correlated to the gel cohesion energy as: ${ }^{45,52}$

$$
E_{c}=\int_{0}^{\gamma_{c}} G_{c}^{\prime} \gamma_{c} d \gamma_{c}=\frac{1}{2} \gamma_{c}^{2} G_{c}^{\prime}
$$

where $E_{c}$ is the cohesion energy and $G^{\prime}{ }_{c}$ is the storage modulus at critical strain. The cohesion energy is a measurement of the 90 energy involved in the formation of physical crosslinks in the network. The results for CHC/SAL gels with different compositions are shown in Table 3. As $\gamma_{c}$ was found to be the same for all investigated formulations, differences in the calculated cohesion energy were directly correlated to differences 95 in shear moduli. Thus the cohesion energies correlate well with the discussion from moduli values that $\mathrm{CHC}$ nanoparticles disturbs the SAL-Ca ${ }^{2+}$ crosslink formation and that Glycerol provides crosslinking hydrogen bonds in the composites.

In summary the CHC/SAL composite hydrogels exhibits many 100 rheological properties making them promising for biomedical application, especially as implantable depot gels. The investigated composites had storage modulus values in the range 10-30 $\mathrm{kPa}$, which should be easy to adjust further by varying parameters such as polymer concentration and gelation medium 105 composition. The moduli are of a similar order as reported values for soft tissue, ${ }^{53-55}$ which has been stated to be an important factor in the host response to implantable devices. ${ }^{5}$ Furthermore, the excellent shear reversibility could allow for direct injection of the gels. Under the large shear during injection the gels would be in a 110 quasi-liquid state and thus exhibit flow. However, after injection 

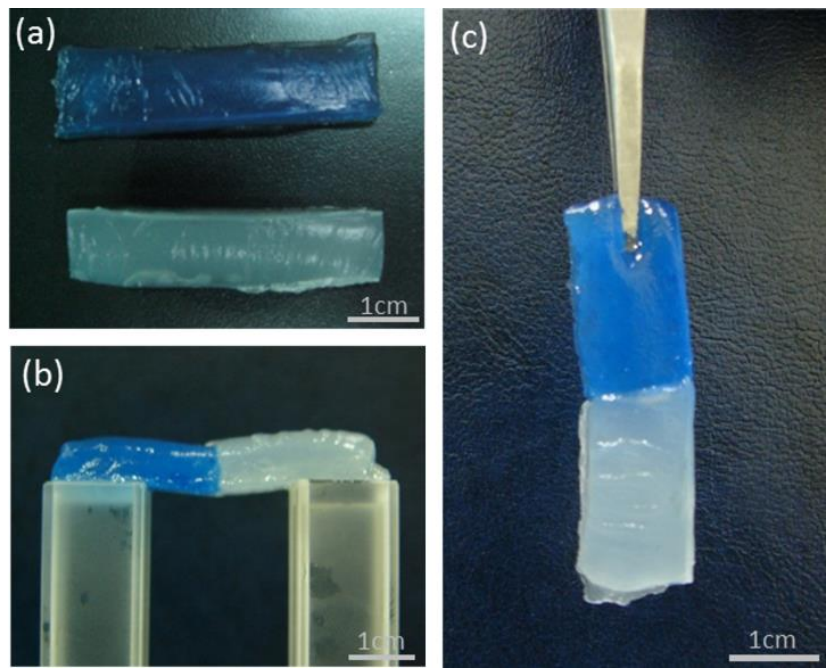

Fig. 2 Photographs illustrating the self-healing properties of CHC/SAL hydrogels. (a) A sample coloured by Trypan blue and a non-coloured sample, both with the weight ratios $\mathrm{CHC} / \mathrm{SAL} / \mathrm{glycerol}=1 / 1 / 10$, prepared 5 by exposure to $2 \% \mathrm{w} / \mathrm{w} \mathrm{CaCl}_{2}$. A bridge constructed by connecting the freshly cut surface of the two samples could be (b) suspended horizontally and (c) held vertically.

the shear forces would be absent and the gels would recover to the original quasi-solid state. ${ }^{32,56-58}$

\section{${ }_{10}$ Self healing}

The ability of a material to self heal damages that occurs during use would be highly desirable for materials that are intended to perform in a designed manner for significant times where repair is not possible. ${ }^{59}$ For hydrogels used in drug delivery the

15 formation of cracks or fractures would increase the surface are through which drug release occurred, leading to increased release, i.e. increased dose per time. In addition, fractures could cause unwanted migration and/or altered degradation of implantable depot gels.

20 Polymer hydrogels formed by covalent bonds are usually brittle and lack the ability to self-heal. ${ }^{60} \mathrm{~A}$ non-covalent approach using dendritic macromolecules as binders in clay nanosheets sodium polyacrylate hydrogels has been reported by Wang et al. ${ }^{61}$ The gels were reported to have high mechanical strength, rapid 25 shear recovery capability and self healing behaviour, as well as a very easy preparation procedure. From the excellent shear recovery displayed by our composite gels, it was reasoned that our dual-structure gels possibly could be self-healing as well. To investigate this, gels were prepared in different compositions. It 30 was found that no self healing was obtained for pure alginate gels with or without glycerol, nor did composite gels without glycerol exhibit self healing behaviour. However, composite gels with high glycerol content could self heal to some extent. This is illustrated in Fig. 2, where the freshly cut surfaces of gels of 35 different colours (blue and translucent, Fig. 2a) with weight ratios $\mathrm{CHC} / \mathrm{SAL} /$ glycerol $=1 / 1 / 10$ have been brought into contact. The healed composite hydrogel was strong enough to hold when suspended horizontally (Fig. 2b) and vertically (Fig. 2c). This self healing could provide increased durability and robustness of the 40 composite gels if used in implant applications.

\section{Equilibrium swelling}

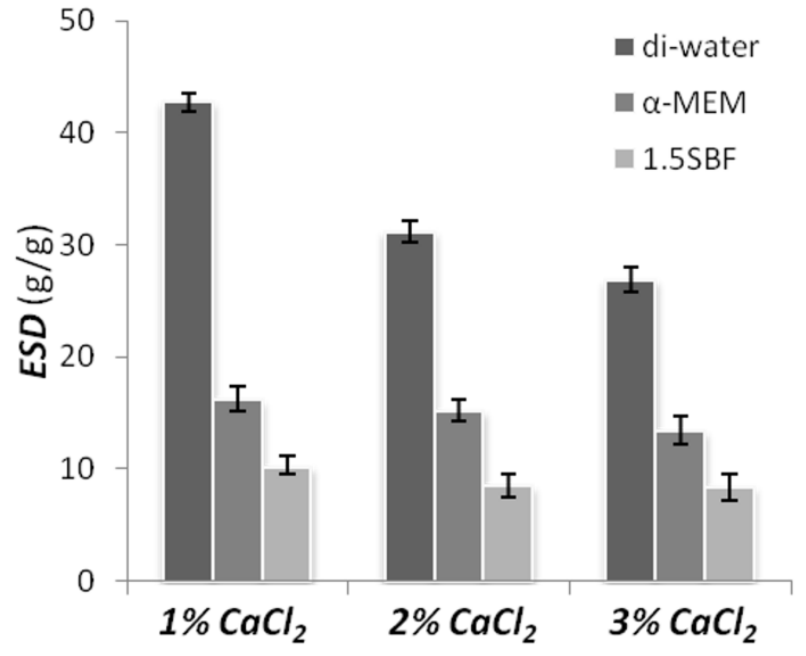

Fig. 3 Equilibrium swelling degree of CHC/SAL composite hydrogels (weight ratio $\mathrm{CHC} / \mathrm{SAL} /$ glycerol $=1 / 1.5 / 0$ ) as a function of $\mathrm{CaCl}_{2}$ concentration in gelation medium. Gels were submerged in di-water, medium ( $\alpha$-MEM) or SBF for 2 days to reach the equilibrium state. Error bars indicate $\operatorname{Min} / \operatorname{Max}(\mathrm{n}=3)$.

Most biological applications are used in a liquid or semi-liquid environment, for swellable materials the swelling is an important 50 material parameter which greatly influences mechanical properties and substance exchange behaviour, i.e. drug release. Thus, the equilibrium swelling degree (ESD) of lyophilized composite hydrogels with the weight ratios $\mathrm{CHC} / \mathrm{SAL} /$ glycerol $=$ $1 / 1.5 / 0$, prepared using gelation media with different $\mathrm{CaCl}_{2}$ 55 concentrations, was determined in deionized water (di-water), cell culture medium $(\alpha-\mathrm{MEM}+10 \%$ FBS $)$, and simulated body fluid (1.5SBF). The extent to which a gel swells is determined by the swelling pressure $(\pi)$, which can be written: ${ }^{62-64}$

$$
\pi=\pi_{\text {mix }}+\pi_{\text {ion }}+\pi_{\mathrm{e}}
$$

60 where $\pi_{\mathrm{mix}}$ is osmotic pressure from the dissolution of polymer chains, $\pi_{\text {ion }}$ is the osmotic pressure derived from counterions within the gel and $\pi_{\mathrm{e}}$ is the elastic pressure derived from the deformation of the polymer network during swelling. ${ }^{62-64}$ The term $\pi_{\mathrm{e}}$ in the above equation is determined by the crosslinking ${ }_{65}$ density, where a high degree of crosslinking corresponds to a high elastic pressure opposing swelling. For the gels in this study the crosslinking should be dominated by the interaction between alginate and $\mathrm{Ca}^{2+}$.

As expected, it was found that in all investigated swelling 70 media the ESD decreased with increasing concentration of $\mathrm{CaCl}_{2}$ in the gelation medium (Fig. 3). Regarding the swelling in different media, the swelling was highest in di-water, less in cell culture medium and the least in 1.5SBF (Fig. 3). The observed values of ESD can be explained by the compositions of the 75 different swelling media. In deionized water the contributions of counterions to the swelling is the highest, i.e. $\pi_{\text {ion }}$ is large. In contrast for the used $\alpha$-MEM with a higher and $1.5 \mathrm{SBF}$ with the highest ionic strength the difference in ion concentration within the gel and in the swelling media is reduced, i.e. $\pi_{\text {ion }}$ decreases. In 80 addition the different compositions of the media could also affect the interaction parameter, which in turn would influence $\pi_{\text {mix }}$. Furthermore, the $\alpha$-MEM and 1.5SBF contains divalent ions 

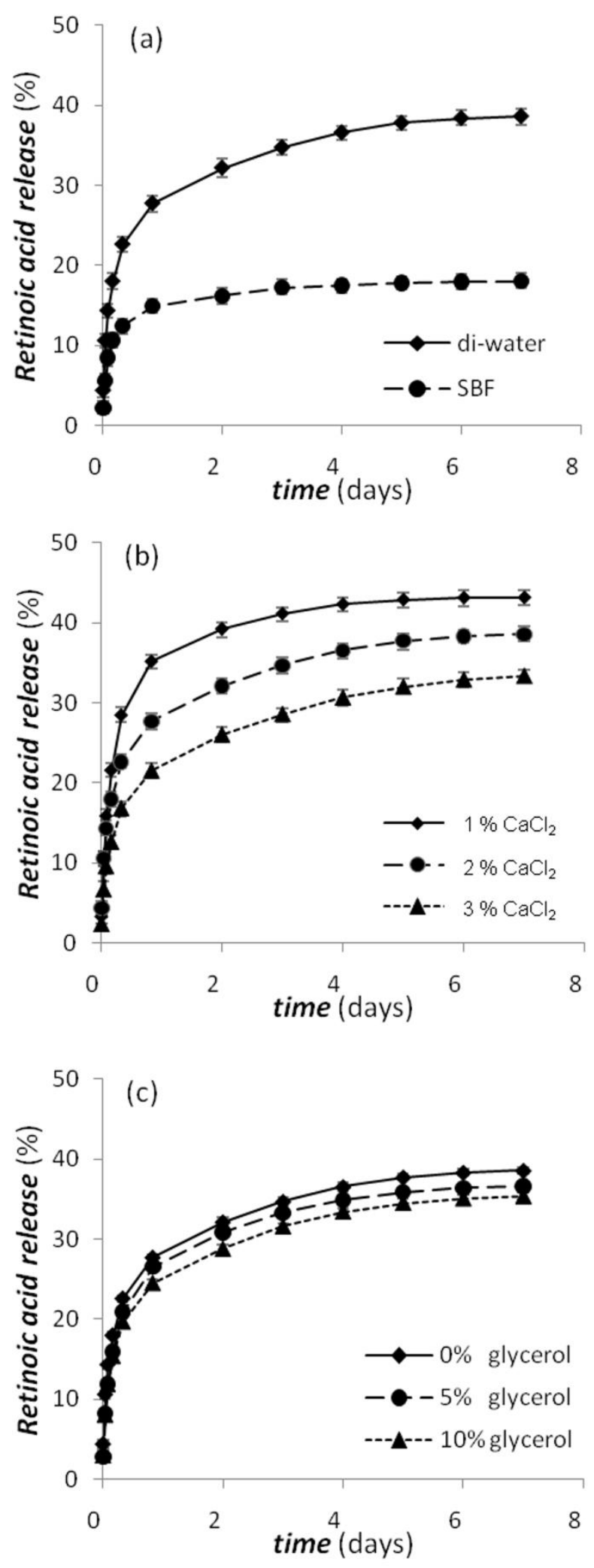

Fig. 4 Release of all-trans retinoic acid encapsulated in $\mathrm{CHC}$ nanoparticles loaded in $\mathrm{CHC} / \mathrm{SAL}$ composite hydrogels with weight ratios $\mathrm{CHC} / \mathrm{SAL}=1 / 1.5$; (a) in di-water and SBF; (b) in di-water with 5 different concentrations of calcium chloride used in the gelation medium;

(c) in di-water with different concentrations of glycerol in the prepared gels. Error bars indicate $\min / \max (n=3)$. which are known to act as crosslinkers in alginate. ${ }^{24}$ Such ions would increase the opposing elastic pressure $\pi_{\mathrm{e}}$ in the above 10 equation, leading to reduced swelling. Calcium ions replacing sodium ions of the SAL would also reduce the number of counterions within the gel due to their divalent charge. This phenomenon with polyvalent ions greatly reducing swelling of oppositely charged polymer gels is well described by ${ }_{15}$ Katchalsky. ${ }^{65}$ The swelling results show that under conditions relevant for applications, the swelling is moderate, indicating that the gels should maintain good structural integrity and mechanical properties.

\section{In Vitro Drug Release}

20 As a model drug in release studies all-trans Retinoic acid was chosen, it is a hydrophobic molecule used in both cancer therapy ${ }^{66}$ and in treatment of dermatological diseases. ${ }^{67}$ As such it is of relevance both for depot implant gels and dermal applications. Release profiles from different preparations of 25 composite hydrogels in di-water and 1.5SBF are shown in Fig. 4. During the one week release study, all investigated samples displayed similar drug release profiles. All samples released only a part of the loaded drug, seemingly reaching a plateau after one week. In Fig. 4a the release data for di-water and $1.5 \mathrm{SBF}$ is 30 shown for hydrogels with the composition of weight ratios $\mathrm{CHC} / \mathrm{SAL} /$ glycerol $=1 / 1.5 / 0$ prepared by exposure to $2 \% \mathrm{w} / \mathrm{w}$ $\mathrm{CaCl}_{2}$. The release of retinoic acid in di-water was much faster than in $1.5 \mathrm{SBF}$ solution. After one week, $39 \%$ of the drug had been released in di-water, to be compared with only $18 \%$ in 35 1.5SBF. The release profiles in di-water for gels prepared using various concentrations of $\mathrm{CaCl}_{2}$ and glycerol is shown in Fig. 4bc. The drug release rate increased somewhat with decreasing $\mathrm{CaCl}_{2}$ concentration in the gelation medium. After one week the released percentage of drug was $43.1 \%$, from gels prepared using $401 \%$ w/w $\mathrm{CaCl}_{2}$ gelation medium, $38.5 \%$ using $2 \%$ w/w $\mathrm{CaCl}_{2}$ and only $33.3 \%$ using $3 \% \mathrm{w} / \mathrm{w} \mathrm{CaCl}_{2}$ (Fig. 4b). For glycerol there was a slight increase in drug release rate with decreasing glycerol content (Fig. 4c). The results demonstrate that the initial release rate of retinoic acid from the $\mathrm{CHC} / \mathrm{SAL}$ composite gels is 45 dominated by the release environment, but also affected by gel composition. To investigate the mechanism of the initial release the release data between 0 and about $60 \%$, as suggested by Ritger and Peppas, ${ }^{68}$ was fitted to the simplified Higuchi equation for systems with the loaded drug in the dissolved state: ${ }^{69}$

50

$$
\frac{M_{t}}{M_{\infty}}=2\left(\frac{D t}{\pi l^{2}}\right)^{0.5}
$$

Where $M_{\mathrm{t}} / M_{\infty}$ is the fraction of drug released, here $100 \%$ release of the investigated process is assumed to have occurred after 7 days, $D$ is the drug diffusion coefficient, $l$ is the initial film thickness, and $t$ is the release time. The release profiles all 55 showed good agreement with diffusion controlled (Fickian release) and from Eq. 6 the diffusion coefficients for the different formulations and conditions were approximated, using a gel thickness of $2 \mathrm{~mm}$ (Table 4). The calculated $D$ values were of the order $10^{-11} \mathrm{~m}^{2} \mathrm{~s}^{-1}$, which is similar to previously reported diffusion ${ }_{60}$ coefficients for dendrimers ${ }^{70}$ and bovine serum albumin ${ }^{71}$ in alginate gels. More specific the calculated $D$ values were all rather similar, but with a slight decrease in $D$ for $3 \% \mathrm{Ca}^{2+}$ in the gelation medium and a significant increase for release in $1.5 \mathrm{SBF}$ 
Table 4 Diffusion coefficients $\left(10^{-11} \mathrm{~m}^{2} \mathrm{~s}^{-1}\right)$ and $\mathrm{R}^{2}$ values for the initial release from samples with different compositions in deionized water or SBF, calculated using Eq. 6 .

\begin{tabular}{cccccccccc}
\hline & \multicolumn{2}{c}{$\begin{array}{c}\text { Release } \\
\text { environment }\end{array}$} & \multicolumn{4}{c}{$\begin{array}{c}\% \mathrm{Ca}^{2+} \text { in } \\
\text { gelation medium }\end{array}$} & \multicolumn{2}{c}{$\begin{array}{c}\text { \% glycerol in } \\
\text { combination } \\
\text { solution }\end{array}$} \\
\hline & di-water & SBF & $1 \%$ & $2 \%$ & $3 \%$ & $0 \%$ & $5 \%$ & $10 \%$ \\
$\mathrm{D}$ & 2.2 & 4.1 & 2.5 & 2.2 & 1.5 & 2.2 & 1.9 & 1.9 \\
$\mathrm{R}^{2}$ & 0.94 & 0.98 & 0.99 & 0.94 & 0.99 & 0.94 & 0.99 & 0.97
\end{tabular}

5 as compared to in di-water.

The partial release of drug and the level off in release after one week, together with the diffusion controlled mechanism of this initial release, indicates that the drug loaded in the composite gels is present in two fractions. (1) Drug loaded in the alginate 10 hydrogel and in the outer shell of the CHC particles, this being the fraction initially released by diffusion through the gel matrix.

(2) Drug loaded in the CHC nanoparticles, the release of this drug fraction would mainly be controlled by the much slower release from the $\mathrm{CHC}$ nanoparticles. The fact that the drug is 15 present in the gels as two fractions is logical. The encapsulation efficiency of the used $\mathrm{CHC}$ is incomplete, and drug loaded $\mathrm{CHC}$ nanoparticles exhibit burst release of a fraction of the drug within one day. ${ }^{36,44,72,73}$ Thus, when the solution with drug loaded CHC was combined with the SAL to form gels, the $15 \%$ of non-loaded 20 drug as well as the fraction released during the initial burst release from the $\mathrm{CHC}$ nanoparticles would be freely available for release from the composite gels.

Interestingly, the amount of drug released in this initial process was found to be dependent on gel composition, but even more on 25 the release environment, as seen in Fig. 4. This means that the fraction of drug available for release through the initial faster process actually changes. This fact suggests that the amount of drug available for fast release can not only be attributed to loading efficiency and burst release from the $\mathrm{CHC}$ nanoparticles. ${ }_{30}$ One likely explanation is that there is a complicated phase behaviour behind the distribution of drug between the phases with fast and slow release, and that this distribution changes with gel composition, swelling and release environment.

The implications of the release characteristics for applications 35 would be that the gels hold potential in long term sustained release, as less than $20 \%$ of the drug is "burst released" in 1.5SBF. However, a simple washing step should be performed prior to injection. For applications where the release should occur over a short time, such as one day, the well defined and 40 reproducible release profiles of up to about $40 \%$ of the loading should also be appealing.

\section{Cytotoxicity evaluation}

To further investigate the potential of the CHC/SAL composites in biomedical applications in-vitro cytotoxicity test were 45 performed. Cells were cultured on $\mathrm{CHC} / \mathrm{SAL}$ gel surfaces and were analyzed using the MTT assay; the results are given in Fig. 5. The cell viabilities were $96 \%, 95 \%$, and $93 \%$ after 24 hours of treatment for pure alginate gels, composite gels and composite gels loaded with retinoic acid at a concentration of $100 \mu \mathrm{g} / \mathrm{ml}$. 50 After two days incubation, the cell survival ratios had decreased for both the pure and drug loaded gels. However, the values of the survival ratios were still above $88 \%$. From the results it can

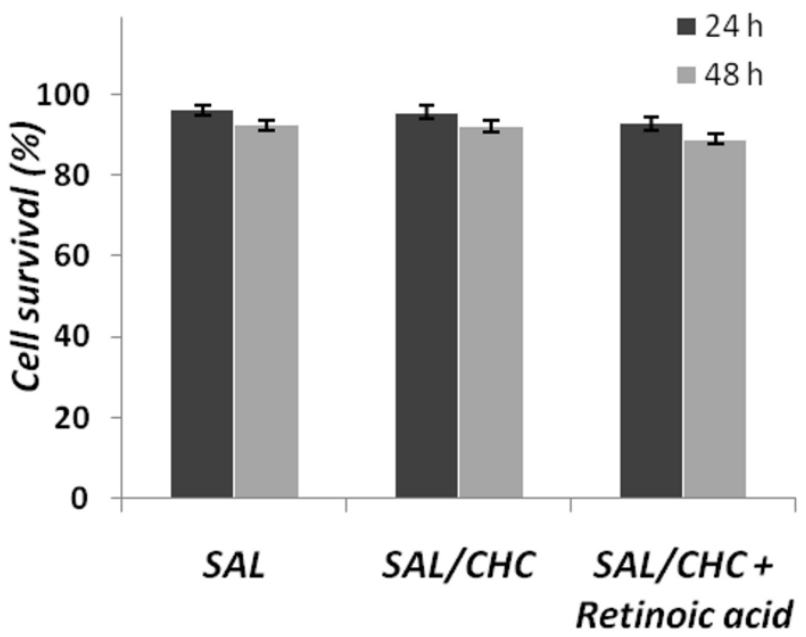

Fig. 5 Human fibroblast survival ratio after incubation for 24 and 48 55 hours on; SAL, CHC/SAL and retinoic acid loaded CHC/SAL hydrogels. The CHC/SAL gels had the weight ratios CHC/SAL/glycerol $=1 / 1.5 / 0$ and the pure SAL gels had a concentration of $1.5 \% \mathrm{w} / \mathrm{w}$. Samples were prepared by exposure to $2 \% \mathrm{w} / \mathrm{w} \mathrm{CaCl}_{2}$ gelation medium. Error bars indicate $\min / \max (\mathrm{n}=8)$.

60 be concluded that native and drug loaded composite gels exhibited low cytotoxicity, similar to pure alginate gels.

\section{In Vivo Primary Irritation Evaluation}

In order to evaluate if the SAL/CHC composite hydrogels caused primary skin irritation, the Draize model was utilized. Gel 65 samples with high and low dose of sodium alginate were investigated using healthy male New Zealand White rabbits. The result revealed that there was no irritation was detected for any of the investigated gel formulations, i.e. the PII values were 0 for composite gels with both low and high dose of SAL. Based on 70 the results of this in vivo investigation, the irritation properties of CHC/SAL composite gels show excellent skin contact properties, holding promises for use in dermal applications.

\section{Conclusions}

A stable sodium alginate - amphiphilic chitosan combination 75 solution was successfully prepared and utilized to form a novel dual-structure hybrid hydrogel, composed of nano-structured $\mathrm{CHC}$ embedded in an alginate matrix. The characterization of the composite gels with regard to rheological properties, swelling, drug delivery, toxicity and skin irritation revealed that the gels 80 held many material properties making them attractive candidates for biomedical applications, such as; high loading of hydrophobic drug, rapid gelling, shear reversibility, well defined drug release, low toxicity etc. Combined, the properties make the $\mathrm{CHC} / \mathrm{SAL}$ gels especially good candidates for use as drug delivery platforms 85 in long term injectable depot gels or dermal applications. Future outlook would be to perform long term release studies and evaluate different therapeutic applications in vivo.

\section{Acknowledgements}

The authors would like to give their thanks to the National 90 Science Council, Taiwan, Republic of China, for the financial support under the project contract NSC 99-2113-M-009-013- 
MY2-3060. The work was performed in collaboration with the VINN Excellence Centre SuMo Biomaterials (Supermolecular Biomaterials - Structure dynamics and properties) and the associated research school BIOSUM.

\section{${ }_{5}$ Notes and references}

${ }^{a}$ Department of Materials Science and Engineering, National Chiao Tung University, HsinChu City, 300 Taiwan. Fax:+886 3 5724727;

Tel:+886 35712121 (ext 55391); E-mail: deanmo_liu@yahoo.ca

${ }^{b}$ Department of Chemical and Biological Engineering, Chalmers

10 University of Technology, 41269 Gothenburg, Sweden. Fax:

+46 31772 3418; Tel: +46 31 7723411; E-mail:

mikael.larsson@chalmers.se

$\dagger$ Electronic Supplementary Information (ESI) available: Tables for the evaluation of primary irritation index. See DOI: 10.1039/b000000x/

$15 \$$ Both authors contributed equally to this work.

1. J.-A. Yang, H. Kim, K. Park and S. K. Hahn, Soft Matter, 2011, 7, 868.

2. M. Malmsten, Soft Matter, 2006, 2, 760.

3. X. Li, X. Kong, J. Zhang, Y. Wang, Y. Wang, S. Shi, G. Guo, F. Luo,

20 X. Zhao, Y. Wei and Z. Qian, J. Pharm. Sci., 2011, 100, 232.

4. S. M. Moghimi, A. C. Hunter and J. C. Murray, Faseb J., 2005, 19, 311.

5. J. Hilborn and L. M. Bjursten, J. Tissue Eng. Regen. Med., 2007, 1, 110.

25 6. P. L. Soo, L. B. Luo, D. Maysinger and A. Eisenberg, Langmuir, 2002, 18, 9996.

7. J. H. Jeong, H. S. Kang, S. R. Yang and J. D. Kim, Polymer, 2003, 44, 583.

8. S. I. Yusa, K. Fukuda, T. Yamamoto, K. Ishihara and Y. Morishima, $30 \quad$ Biomacromolecules, 2005, 6, 663.

9. X. Z. Qu, V. V. Khutoryanskiy, A. Stewart, S. Rahman, B. Papahadjopoulos-Sternberg, C. Dufes, D. McCarthy, C. G. Wilson, R. Lyons, K. C. Carter, A. Schatzlein and I. F. Uchegbu, Biomacromolecules, 2006, 7, 3452.

35 10. L. Sheihet, R. A. Dubin, D. Devore and J. Kohn, Biomacromolecules, 2005, 6, 2726.

11. M. L. Gou, X. Y. Li, M. Dai, C. Y. Gong, X. H. Wang, Y. Xie, H. X. Deng, L. J. Chen, X. Zhao, Z. Y. Qian and Y. Q. Wei, Int. J. Pharm., 2008, 359, 228.

40 12. J. M. Barichello, M. Morishita, K. Takayama and T. Nagai, Int. J. Pharm., 1999, 184, 189.

13. X. Z. Zhang, P. J. Lewis and C. C. Chu, Biomaterials, 2005, 26, 3299.

14. P. J. Flory, Principles of Polymer Chemistry, Cornell University 45

15. T. S. Chow, J. Mater. Sci., 1980, 15, 1873.

16. R. K. Richardson, G. Robinson, S. B. Rossmurphy and S. Todd, Polym. Bull., 1981, 4, 541.

17. T. S. Chow, J. Polym. Sci. Polym. Phys., 1982, $20,2103$.

50 18. T. Vanvliet, Colloid Polym. Sci., 1988, 266, 518.

19. D. W. Schaefer and R. S. Justice, Macromolecules, 2007, 40, 8501.

20. M. Larsson, M. Stading and A. Larsson, Soft Mater., 2010, 8, 207.

21. J. L. Valentín, I. Mora-Barrantes, J. Carretero-González, M. A. López-Manchado, P. Sotta, D. R. Long and K. Saalwächter,

$55 \quad$ Macromolecules, 2009, 43, 334.

22. J. Zhang, C. R. Daubert, J. H. Mulligan and E. A. Foegeding, J. Texture Stud., 2008, 39, 582.
23. J. H. Zhang, C. R. Daubert and E. A. Foegeding, J. Texture Stud., 2006, 37, 200.

60 24. M. George and T. E. Abraham, J. Control. Release, 2006, 114, 1.

25. M. Hamidi, A. Azadi and P. Rafiei, Adv. Drug Deliv. Rev., 2008, 60, 1638.

26. J. H. Zhang, C. R. Daubert and E. A. Foegeding, J. Food Sci., 2005, 70, E425.

65 27. K. H. Liu, S. Y. Chen, D. M. Liu and T. Y. Liu, Macromolecules, 2008, 41, 6511.

28. T. Y. Liu, S. Y. Chen, Y. L. Lin and D. M. Liu, Langmuir, 2006, 22, 9740.

29. L. S. Nair, T. Starnes, J.-W. K. Ko and C. T. Laurencin, $70 \quad$ Biomacromolecules, 2007, 8, 3779.

30. X. Li, X. Kong, X. Wang, S. Shi, G. Guo, F. Luo, X. Zhao, Y. Wei and Z. Qian, Eur. J. Pharm. Biopharm., 2010, 75, 388.

31. S. K. Motwani, S. Chopra, S. Talegaonkar, K. Kohl, F. J. Ahmad and R. K. Khar, Eur. J. Pharm. Biopharm., 2008, 68, 513.

75 32. H. Park, S. W. Kang, B. S. Kim, D. J. Mooney and K. Y. Lee, Macromol. Biosci., 2009, 9, 895.

33. D. R. Picout, R. K. Richardson, C. Rolin, R. M. Abeysekera and E. R. Morris, Carbohydr. Polym., 2000, 43, 113.

34. B. Dupuy, A. Arien and A. P. Minnot, Artif. Cells Blood Substit. $80 \quad$ Immobil. Biotechnol., 1994, 22, 71.

35. A. Haug, B. Larsen and O. Smidsrød, Carbohyd. Res., 1974, 32, 217.

36. K.-H. Liu, B.-R. Chen, S.-Y. Chen and D.-M. Liu, J. Phys. Chem. B, 2009, 113, 11800.

37. R. Jin, L. S. M. Teixeira, P. J. Dijkstra, M. Karperien, C. A. van 85 Blitterswijk, Z. Y. Zhong and J. Feijen, Biomaterials, 2009, 30, 2544.

38. S. Kim, S. K. Nishimoto, J. D. Bumgardner, W. O. Haggard, M. W. Gaber and Y. Z. Yang, Biomaterials, 2010, 31, 4157.

39. M. Assad, A. Chernyshov, M. A. Leroux and C. H. Rivard, Bio-Med.

$90 \quad$ Mater. Eng., 2002, 12, 339.

40. S. Golla, S. Madihally, R. L. Robinson and K. A. M. Gasem, Toxicol. Vitro, 2009, 23, 176.

41. G. Mazzanti, C. Daniele, B. Tita, F. Vitali and A. Signore, Dent. Mater., 2005, 21, 371.

95 42. J. H. Draize, G. Woodard and H. O. Calvery, J. Pharmacol. Exp. Ther., 1944, 82, 377.

43. N. A. Abdulmola, M. W. N. Hember, R. K. Richardson and E. R. Morris, Carbohydr. Polym., 1996, 31, 65.

44. K.-H. Liu, S.-Y. Chen, D.-M. Liu and T.-Y. Liu, Macromolecules, $100 \quad 2008,41,6511$

45. J. Y. Cho, M. C. Heuzey, A. Begin and P. J. Carreau, Biomacromolecules, 2005, 6, 3267.

46. S. J. Rendevski and A. N. Andonovski, Polym. Bull., 2005, 54, 93.

47. L. R. G. TRELOAR, The Physics of Rubber Elasticity, 3rd Ed. edn., $105 \quad$ Clarendon Press, Oxford, U.K., 1975.

48. J. B. GluckHirsch and J. L. Kokini, J. Rheol., 1997, 41, 129.

49. J. R. Mitchell, J. Texture Stud., 1980, 11, 315.

50. A. Segeren, J. Boskamp and M. Van Den Tempel, Faraday discuss., 1974, 57, 255.

110 51. G. Kraus, J. Appl. Polym. Sci., 1963, 7, 861.

52. D. G. Lessard, M. Ousalem, X. X. Zhu, A. Eisenberg and P. J. Carreau, J. Polym. Sci. Polym. Phys., 2003, 41, 1627. 
53. E. Sapine-de Brosses, J. L Gennisson, M. Pernot, M. Fink andM. Tanter, Phys. Med. Biol., 2010, 55, 1701.

54. E. J. Chen, J. Novakofski, W. K. Jenkins and W. D. O'Brien, Jr., IEEE Trans. Ultrason. Ferroelectr., Freq. Control, 1996, 43, 191.

5

55. X. Li, G. Wang, L. Huang and G. Zhang, Instrum. Sci. Technol., 2006, 34, 393

56. R. Barbucci, D. Pasqui, R. Favaloro and G. Panariello, Carbohyd. Res., 2008, 343, 3058.

10 57. M. Dolz, M. J. Hernandez, J. Pellicer and J. Delegido, J. Pharm. Sci., 1995, 84, 728.

58. N. Markovic, N. K. Dutta, D. R. G. Williams and J. Matisons, in Polymer Gels: Fundamentals and Applications, eds. H. B. Bohidar, P. Dubin and Y. Osada, Amer Chemical Soc,

15 Washington, 2003, vol. 833, pp. 190.

59. R. P. Wool, Soft Matter, 2008, 4, 400.

60. N. A. Peppas, Y. Huang, M. Torres-Lugo, J. H. Ward and J. Zhang, Аnnu. Rev. Biomed. Eng., 2000, $2,9$.

61. Q. Wang, J. L. Mynar, M. Yoshida, E. Lee, M. Lee, K. Okuro, K.

$20 \quad$ Kinbara and T. Aida, Nature, 2010, 463, 339.

62. S. T. Moe, G. Skjaak-Braek, A. Elgsaeter and O. Smidsroed, Macromolecules, 1993, 26, 3580.

63. M. Larsson, S. Gustafsson, E. Olsson and A. Larsson, e-Polymers, 2009, vol.:141.

25 64. F. Horkay, I. Tasaki and P. J. Basser, Biomacromolecules, 2000, 1, 84.

65. A. Katchalsky, Biophys. J., 1964, 4, 9.

66. A. Jimenez-Lara, A. Aranda and H. Gronemeyer, Molecular Cancer, $2010,9,15$.

30 67. V. C. O. Njar, L. Gediya, P. Purushottamachar, P. Chopra, T. S. Vasaitis, A. Khandelwal, J. Mehta, C. Huynh, A. Belosay and J. Patel, Biorg. Med. Chem., 2006, 14, 4323.

68. P. Ritger and N. Peppas, J. Control. Release, 1987, 5, 37.

69. W. I. Higuchi, J. Pharm. Sci., 1962, 51, 802.

35 70. D. Bernin, G. J. Goudappel, M. van Ruijven, A. Altskär, A. Ström, M. Rudemo, A. M. Hermansson and M. Nydén, Soft matter, accepted for publication, DOI:10.1039/C1SM05070B.

71. B. Amsden and N. Turner, Biotechnol. Bioeng., 1999, 65, 605.

72. T.-Y. Liu and Y.-L. Lin, Acta Biomaterialia, 2010, 6, 1423.

40 73. T.-Y. Liu, S.-Y. Chen, Y.-L. Lin and D.-M. Liu, Langmuir, 2006, 22 , 9740 . 